\title{
Postoperative Complications of Inguinal Hernia Surgery
}

*Ashrafuzzaman $\mathrm{M}^{1}$, Bhattacharjee $\mathrm{A}^{2}$

\begin{abstract}
Inguinal hernia surgery is a common operation. It has some negative deviation in its postoperative course. Some immediate and chronic complication can make the doom like situation for both patients and surgeons. Regarding the etiology of these complications; debate still exists. Two main objectives of the study was to determine the frequency of postoperative complications of inguinal hernia surgery as well as their level of severity. The study was conducted among the patients admitted at department of Surgery in Rajbari District Hospital. The study period was 24 months; started from January 2015 to December 2016. The patients were enrolled by purposive sampling. A pre-structured, peer-reviewed data collection sheet was used as a tool to record data regarding socio-demographic clinical, surgical and post operative profile. After taking informed written consent all patients were sent for the pre-anesthetic check up. Lichtenstein tension free mesh hernioplasty was done by consultant surgeon. Injection ceftriaxone single dose $i / v$ was used as prophylactic antibiotic. Here in all cases prolene mesh was used manufactured by Jhonson \& Jhonson. Data were compiled, edited, analyzed and plotted into tabular and figure form. Out of 124 patients, $37.09 \%$ from $21-30$ years age group whereas $29.83 \%$ from $31-40$ years age group. The mean age was $28.79 \pm 7.16$ years (age range: $17-69$ years). Out of 124 patients $34.67 \%$ were day laborer, $23.39 \%$ were transport laborer and $16.93 \%$ were service holder. Among them $26.61 \%$ patients admitted as emergency cases and rest $73.38 \%$ was admitted as routine cases. After surgery, 26(20.97\%) patients experienced post operative complication. Among them 8(30.76\%), 6(23.07\%) and
\end{abstract}

1. ${ }^{*}$ Dr. Md. Ashrafuzzaman, MBBS, MS (Surgery). Senior consultant (Surgery), Razbari District Hospital, Razbari. Call: 01912-656814

2. Dr. Avisak Bhattacharjee MBBS, FCPS (Surgery), FMAS (India), MPH (Epidemiology), PhD Research Fellow; Department of Surgical Oncology, NICRH, Mohakhali, Dhaka-1212.

*For Correspondence
12(46.15\%) had early, medium and late complications respectively. Besides, 12(46.15\%) were categorized as Clavien-Dindo Grade-I whereas 10(38.46\%) were categorized as Grade-II 2(7.69\%) patients each were categorized as IIIa and IIIb. Emergency admission (46.15\%) was the major risk factor. Higher ASA grade, emergency admission, pre-existing pain, severe depression, pre-existing uncontrolled DM and some drugs as such as clopidogrel and aspirin are the significant preoperative risk factors. Preexisting uncontrolled DM and drug effects can not be corrected in case of emergency surgery.

Keywords: Inguinal Hernia, Hernioplasty.

\section{INTRODUCTION}

Inguinal hernia repair is one of the commonest surgeries throughout the world. Approximately 20 million inguinal hernia surgery are performed each year worldwide. ${ }^{1}$ In the "pre-mesh" era, recurrence rate was the single most vital endpoint. After introduction of mesh use in routine hernia surgery in 1980's, the threshold of "acceptable" recurrence rate has been slashed considerably. In 1990's several risk factors were recognized that are responsible for chronic pain. Since then chronic pain after inguinal hernia surgery popularly termed as 'inguinodynia' has drawn the notice of surgeons. ${ }^{2}$ However, laparoscopic techniques are associated with lower risk of inguinodynia. ${ }^{3}$ Inguinodynia is nothing but an experience and as such subjective feeling that compels a person underwent inguinal surgery recently to seek medical attention. ${ }^{4}$ This condition may happen in $0.7-36.7 \%$ cases $^{5}$ and among them $3 \%$ people experience severe inguinodynia. ${ }^{5,6}$ Complications of inguinal hernia surgery may be co notated as an uncommon course of disease accompanied by particular symptoms. Some underlying illness and/or the treatment of illness may be responsible for developing the complications. The severity of complications may be classified according to Clavien-Dindo Classification Scale. ${ }^{7}$ It is a 7 point scale that can be used for surgical complication severity that was first proposed in early 2000.

Besides, only hernia surgery complications can be classified as early, medium and late. Pain, bleeding, urinary retention, 
anesthesia related complications can be discussed under 'early complications'. Seroma and wound infection can be categorized as 'medium complications'. Chronic pain (inguinodynia) and testicular atrophy can be exemplified as 'late complications'. ${ }^{8}$

Major wound infection is fortunately an infrequent occurrence as hernia surgery is a clean surgery. It is more common in case of strangulated inguinal hernia. Obesity, prolonged duration of operation and use of local anesthesia are the common contributing factors for wound infection. Improper hemostasis and inadvertent surgery are responsible for seroma and hematoma. These early complications may also lead to wound infection. ${ }^{8}$ Postoperative hydrocele may occur due to redundant large portion of sac that is kept in situ. ${ }^{9}$ The systemic complications were beyond scope of this research.

The aim of this study was to determine the frequency of postoperative complications of inguinal hernia surgery as well as their level of severity.

\section{MATERIALS AND METHODS}

The study was conducted at the department of Surgery in Rajbari District Hospital. All the patients underwent inguinal hernia repair were enrolled in the study by purposive sampling. The study period was 24 months started from January 2015 to December, 2016. All patients were explained regarding the surgery and the research. After taking informed written consent the patients were sent to the department of Anesthesia with necessary investigations for the pre-anesthetic check up. Here the patients underwent Lichtenstein tension free mesh hernioplasty under spinal anesthesia. All the patients received single dose injectable antibiotic Ceftriaxone. The prolene mesh manufactured by Jhonson and Jhonson was used for every patient. A pre-tested, peer reviewed, interview and observation based data collection sheet was prepared. Data regarding socio-demographic, clinical, operative and post operative profiles were recorded. Data were compiled, edited, managed and analyzed. The mean of age, duration of symptoms were determined. Chi-square test and student's $t$ test were done for data analysis in case of categorical and quantitative values respectively. The $\mathrm{p}$ value was significant at $<0.05$. All data entry and analysis were done with the help of statistical package for social science (SPSS) version 23.

\section{RESULTS}

Out of 124 patients $37.09 \%$ from age group 21-30 years, $29.83 \%$ were from $31-40$ years age group and $15.32 \%$ were from $<20$ years age group. The mean age of the patients was $28.79 \pm 7.16$ years (mean age: $17-69$ years). Only $6(4.83 \%)$ patients were female among 124 patients. Rest 118(95.16\%) were male. The male to female ratio was 19.6:1.

Table-I: Patient's demographic and baseline characteristics $(n=124)$

\begin{tabular}{|l|r|}
\hline Demographic \& Baseline characteristics & Values \\
\hline Age distribution & $19(15.32 \%)$ \\
\hline$<20$ & $46(37.09 \%)$ \\
\hline $21-30$ & $37(29.83 \%)$ \\
\hline $31-40$ & $5(4.03 \%)$ \\
\hline $41-50$ & $17(13.7 \%)$ \\
\hline$>50$ & $28.79 \pm 7.16$ \\
\hline Mean age \pm SD (in years) & $17-69$ \\
\hline Age range (in years) & \\
\hline Sex distribution & $118(95.16 \%)$ \\
\hline Male & $6(4.83 \%)$ \\
\hline Female & $19.6: 1$ \\
\hline Sex ration M:F & \\
\hline Occupation status & $43(34.67 \%)$ \\
\hline Day labourer & $29(23.39 \%)$ \\
\hline Transport worker & $21(16.93 \%)$ \\
\hline Service holder & $16(12.9 \%)$ \\
\hline Business & $15(12.09 \%)$ \\
\hline Others & \\
\hline
\end{tabular}

Out of 124 patients $34.67 \%$ were day labourer $23.39 \%$ were transport worker, $16.93 \%$ were service holder, $12.9 \%$ were businessperson and rest $12.09 \%$ were included as 'others'. Here 'others' denotes the unemployed, retired persons, students and housewife's.

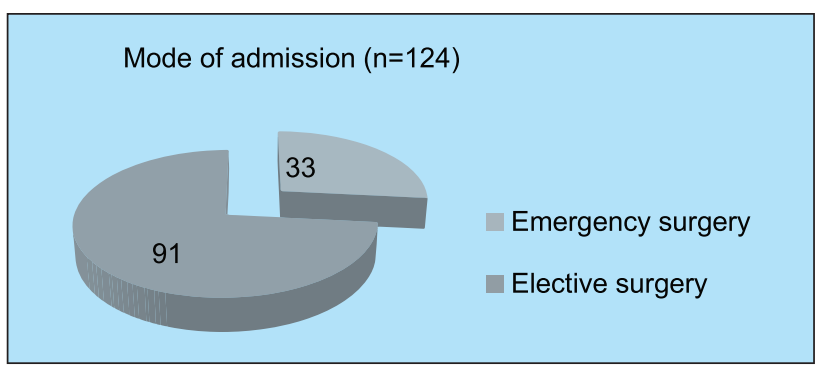

Figure-1: Distribution of patients according to mode of admission $(n=124)$ 
Out of 124 patients $33(26.61 \%)$ patients admitted as emergency patients whereas rest 91(73.38\%) underwent surgery as elective cases.

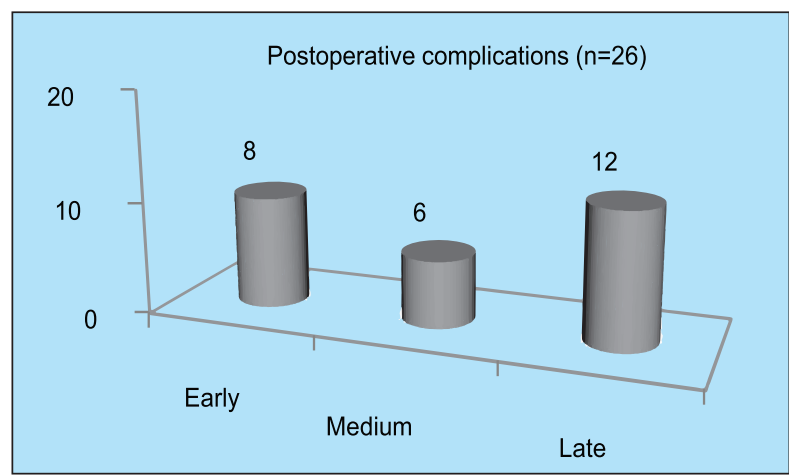

**2 patients experienced early complications followed by medium complication.

Figure-2: Distribution of patients according to postoperative complications $(n=26)$

Out of 124 patients only 26(20.97\%) experienced postoperative complications. Among them $8(30.76 \%)$ had early complications, 6(23.07\%) had medium complications and $12(46.15 \%)$ had late complications.

Table-II: Distribution of patients according to specific complications $(n=26)$

\begin{tabular}{|l|c|}
\hline $\begin{array}{l}\text { Complications } \\
\text { Early }(\mathrm{n}=8)\end{array}$ & $\begin{array}{c}\text { Frequency }(\%) \\
(\mathrm{n}=26)\end{array}$ \\
\hline Post spinal headache & $3(11.53 \%)$ \\
Hematoma & $1(3.84 \%)$ \\
Urinary retention & $4(15.38 \%)$ \\
Medium wound infection & $2(7.69 \%)$ \\
Seroma & $4(15.38 \%)$ \\
Late inguinodynia & $10(38.46 \%)$ \\
Testicular atrophy & $1(3.84 \%)$ \\
Postoeprative Hydrocele & $1(3.84 \%)$ \\
\hline
\end{tabular}

Out of 26 patients $38.46 \%$ experienced inguinodynia, $15.38 \%$ had urinary retention and seroma each. $11.53 \%$ patients had early pain, post spinal headache each. Here 2 patients who experienced hematoma and post spinal headache in early period also had wound infection and seroma in later period respectively.

Grading according to Clavien -Dindo classification

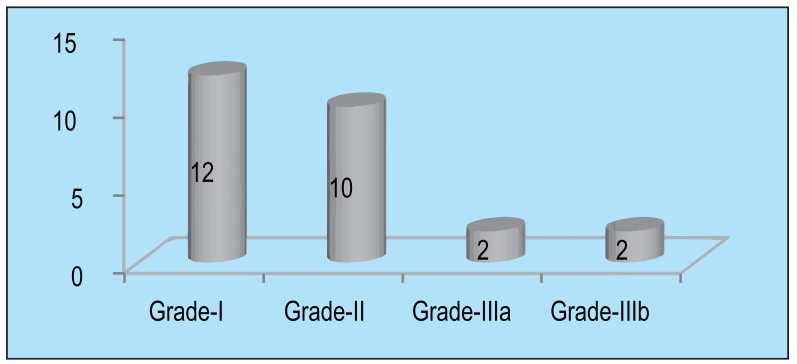

Figure-3: Distribution of patients according to

Clavien-Dindo classification $(\mathrm{n}=124)$

Out of 26 patients $12(46.15 \%)$ were categorized as GradeI, $10(38.46 \%)$ were categorized as Grade-III and 2 (7.69\%) were categorized as Grade-IIIa and IIIb each according to Clavien-Dindo classification of post operative complications of inguinal hernia surgery.

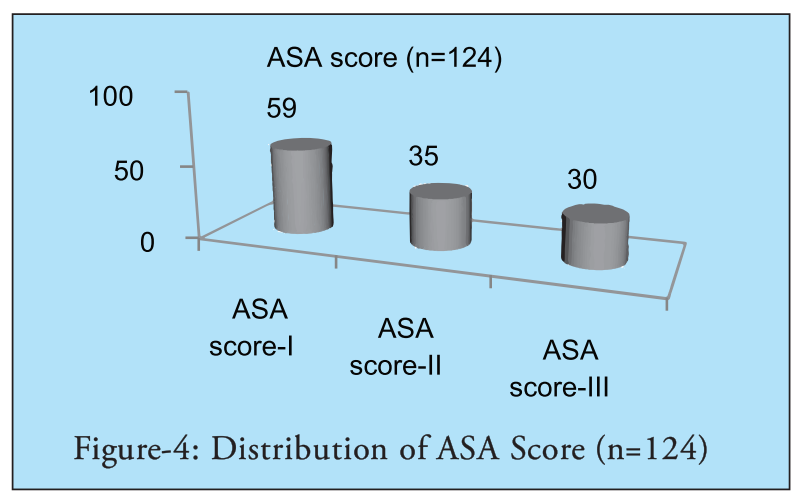

ASA score I was graded in case of 59(47.58\%) patients, II was graded in $35(28.22 \%)$ cases and III was graded in $30(24.19 \%)$ patients.

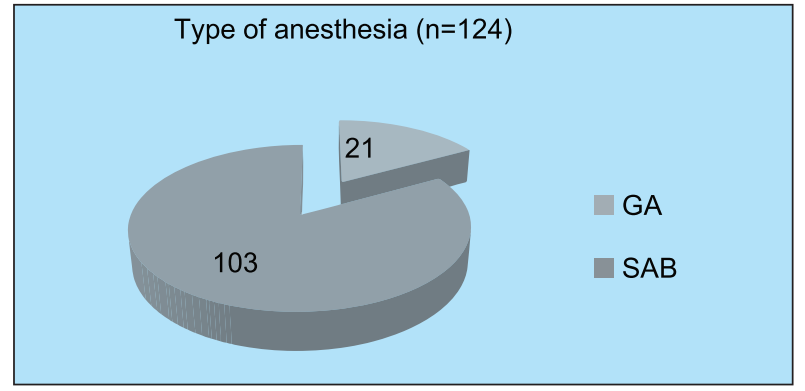

Figure-5: Distribution of patients according to type of anesthesia $(n=124)$ 
Out of 124 patients $103(83.06 \%)$ underwent surgery under subarachnoid block and rest 21(16.93\%) patients underwent surgery under general anesthesia due to some systemic limitations.

Table-III: Distribution of risk factors in complicated cases $(n=26)$

\begin{tabular}{|l|c|}
\hline Risk factors & Frequency (\%) \\
\hline Early age (<50) & $11(42.30 \%)$ \\
\hline ASA grade III & $8(30.76 \%)$ \\
\hline Emergency admission & $12(46.15 \%)$ \\
\hline General anesthesia & $6(23.07 \%)$ \\
\hline Pre-existing pain & $4(15.38 \%)$ \\
\hline Severe depression & $3(11.54 \%)$ \\
\hline Drug-Clopidogrel, Aspirin & $1(3.85 \%)$ \\
\hline Pre-existing uncontrolled DM & $1(3.85 \%)$ \\
\hline
\end{tabular}

*ASA-American Society of Anesthesiology

*In a few cases multiple risk factors were present.

Out of 26 patients experienced complications $46.15 \%$ admitted as emergency cases, $42.30 \%$ low age, manifested $30.76 \%$ were ASA grade III and $23.07 \%$ underwent surgery by general anesthesia.

\section{DISCUSSION}

Post operative complications after inguinal hernia surgery is a bitter experience for general surgeons. The multiple risk factors are responsible for the versatile dimension of post operative complications. Early age as a risk factor for postoperative pain is well known. ${ }^{10}$ In our study, the mean age of the patients was $28.79 \pm 7.16$ years (age range: 17-69 years). We have identified 26 complications of different verities in our study. Among these 26 patients, 11(42.30\%) had the age below 50 years. Henceforth, our study results were partially agreed by a similar previous study. ${ }^{11}$

Nilsson et al. ${ }^{12}$ stated that the most relevant risk factors for 30 day morbidity after hernia repair are localization of hernia (femoral vs inguinal), patient age, ASA score, mode of admission (emergency vs elective surgery) and type of anesthesia. Our study was conducted upon only inguinal hernia surgery. As a result, Nilsson et al first risk factor is excluded. It was mentioned before the low age is a risk factor which was also agreed by Nilsson et al. ${ }^{12}$
Here, we observed $30.76 \%$ cases were ASA grade III who suffered from post operative complications.

Post operative chronic pain is a distressful condition for patient. Here the second victim is the surgeon as because the patient always communicates to him for getting relief. This pain causes social disability, interferes walking, standing and sitting too. Nielsen and Poobalan reported $11.3 \%$ and $14.2 \%$ patients respectively with social disability in their studies. ${ }^{11,13}$ On the contrary, Condon ${ }^{14}$ disagreed with their findings as he reported that chronic pain may not occur more than $1 \%$. This study reports $38.46 \%$ of total post operative complications was chronic pain which was supported by Nielsen and Poobalan's report. ${ }^{11,12}$

Courtney et al. ${ }^{15}$ finds that a high level of preoperative pain indicates an increased risk of long term post operative pain. In this study, we have found that $15.38 \%$ of total complications population had this sort of pre-existing pain. This might suggest that the hernia disease was already complicated prior to surgery in some patients, stretching, entrapment, and/or inflammation of local nerves are conceivable mechanisms, but psychological susceptibility or increased pain sensitivity may also play a role. Moreover, the pain prior to the operation may also have originated from other conditions than the hernia and will then persist after the operation. There is another possibility that is inter individual variations in the manner of communicating subjective feelings may have affected the observed relationship.

In this study we have recognized post spinal headache, hematoma, urinary retention and acute pain as early complications; wound infection and seroma as medium complications and inguinodynia and testicular atrophy as late complications.

Another post surgical complication classification was followed here. This classification is popularly known as Clavien-Dindo classification. This classification system has come from the backdrop of negative outcome of surgery.

Every surgery may have a positive and a negative outcome. Negative outcome consists of complications, failure to cure and sequele ${ }^{16}$. Complications may be defined as any deviation from the normal postoperative course. The complications of surgery was re-write by Daniel Dindo and his colleagues in $2004^{7}$ that was termed as Clavien-Dindo classification. In our study, we have reported only grade I, II, IIIa and IIIb of this classification. 
Table-IV: Classification of Surgical Complications ${ }^{7}$

\begin{tabular}{|c|c|}
\hline Grade & Definition \\
\hline Grade I & $\begin{array}{l}\text { Any deviation from the normal postoperative course without the need for pharmacological } \\
\text { treatment or surgical, endoscopic, and radiological interventionsAllowed therapeutic regimens } \\
\text { are: drugs as antiemetics, antipyretics, analgetics, diuretics, electrolytes, and physiotherapy. This } \\
\text { grade also includes wound infections opened at the bedside }\end{array}$ \\
\hline Grade II & $\begin{array}{l}\text { Requiring pharmacological treatment with drugs other than such allowed for grade I } \\
\text { complicationsBlood transfusions and total parenteral nutrition are also included }\end{array}$ \\
\hline Grade III & Requiring surgical, endoscopic or radiological intervention \\
\hline Grade IIIa & Intervention not under general anesthesia \\
\hline Grade IIIb & Intervention under general anesthesia \\
\hline Grade IV & Life-threatening complication (including CNS complications)* requiring ICU management \\
\hline Grade IVa & Single organ dysfunction (including dialysis) \\
\hline Grade IVb & Multiorgan dysfunction \\
\hline Grade V & Death of a patient \\
\hline Suffix "d" & $\begin{array}{l}\text { If the patient suffers from a complication at the time of discharge (see examples in Table 2), the } \\
\text { suffix "d" (for "disability") is added to the respective grade of complication. This label indicates } \\
\text { the need for a follow-up to fully evaluate the complication. }\end{array}$ \\
\hline
\end{tabular}

*Brain hemorrhage, ischemic stroke, subarrachnoidal bleeding, but excluding transient ischemic attacks.CNS, central nervous system; IC, intermediate care; ICU, intensive care unit.

According to our study, seroma and hematoma required intervention that was not under general anesthesia (IIIa). Hematoma was found in the patient who underwent emergency surgery and was a common user of clopidrogel and aspirin. For chronic pain and wound infection, we had to intervene 2 cases under general anesthesia (IIIb). In infected case, we had to remove mesh under general anesthesia. Rest of the cases were treated with or without pharmacological agents. It is noteworthy here that some pharmacological agents are allowed in grade I we have got $46.15 \%$ grade I, $38.46 \%$ grade II and $7.69 \%$ grade IIa and b each. In our context, $84.6 \%$ (Clavien-Dindo grade I and II) were manageable by conservative means. In the other part of coin it was observed that $15.39 \%$ required intervention (Clavien-Dindo grade $\geq \mathrm{III}$ ). It was almost agreed by Dirk Weyhe and his colleagues. ${ }^{1}$

In a district hospital of Bangladesh it is very tough to provide quality surgery due to the limitations of versatile logistic supports. It was tried up to the level best to perform quality surgery here. But though we had to admit some post surgical complications in case of inguinal hernia repair of which statistics were world standard. Like other settings, chronic pain here has cut the maximum figure as complication.

\section{CONCLUSIONS}

Higher ASA grade, emergency admission, pre-existing pain, severe depression, pre-existing uncontrolled DM and some drugs as such as clopidrogel and aspirin are the significant preoperative risk factors in this study.

\section{REFERENCES}

1. Weyhe D, Tabriz N, Sahlmann B, Verena-Nicole Uslar. Risk factors for perioperative complications in inguinal hernia repair - a systematic review. Innov Surg Sci 2017; aop.

2. Franneby U, Sandblom G, Nordin P, Nyren O, Gunnarsson U. Risk factors for long-term pain after hernia surgery. Annals of surgery, 2006;244(2):212-9.

3. Eklund A, Montgomery A, Bergkvist L, Rudberg C. Lichtenstein inguinal hernia repair. The British journal of surgery, 2010;97(4):600-8.

4. The International Association for the Study of Pain http://www.iasp-pain.org/: the International Association for the Study of Pain [cited 2017 201702-20].

5. Kingsnorth AN, Bowley DM, Porter C. A prospective study of 1000 hernias: results of the Plymouth Hernia Service. Ann R Coll Surg Engl 2003; 85: 18-22. 
6. Bay-Nielsen M, Perkins FM, Kehlet $\mathrm{H}$ and Danish Hernia Database. Pain and functional impairment 1 year after inguinal herniorrhaphy: a nationwide questionnaire study. Ann Surg 2001; 233: 1-7.

7. Dindo D, Demartines N, Clavien P-A. Classification of surgical complications: a new proposal with evaluation in a cohort of 6336 patients and results of a survey. Ann Surg 2004;240:205-13.

8. Norma SW, P. Ronan OC, Andrew WM. Bailey \& Love's-Short practice of surgery. $27^{\text {th }}$ edn. CRC Press (Taylor \& Francis Group); 2018;1034.p.

9. Ray D. Gaines. Complications of Groin Hernia Repair: Their Prevention and Management. Journal of the National Medical Association, vol. 70, no. 3, 1978.

10. Nienhuijs, S.W., et al., Randomized trial comparing the Prolene Hernia System, mesh plug repair and Lichtenstein method for open inguinal hernia repair. Br J Surg, 2005. 92(1): p. 33-8.
11. Poobalan, A.S., et al., Chronic pain and quality of life following open inguinal hernia repair. Br J Surg, 2001. 88(8): p. $1122-26$

12. Nilsson H, Angeras U, Sandblom G, Nordin P. Serious adverse events within 30 days of groin hernia surgery. Hernia 2016;20:377-85.

13. Bay-Nielsen M, Perkins FM, Kehlet H. Pain and functional impairment 1 year after inguinal herniorrhaphy: a nationwide questionnaire study. Ann Surg. 2001;233:1-7.

14. Condon RE. Groin pain after hernia repair. Ann Surg. 2001;233:8.

15. Courtney CA, Duffy K, Serpell MG, et al. Outcome of patients with severe chronic pain following repair of groin hernia. Br J Surg. 2002; 89:1310-1314.

16. Clavien P, Sanabria J, Strasberg S. Proposed classification of complication of surgery with examples of utility in cholecystectomy. Surgery. 1992;111:51826. 